\title{
Las divisiones administrativas en las ciudades españolas, problemas que plantea su heterogeneidad, necesidad de un replanteamiento
}

M. a Jose Aguilera Arilla y M. ${ }^{a}$ Pilar González Yanci *

\section{INTRODUCCION}

Es bien sabido que el geógrafo al realizar su investigación espacial encuentra múltiples dificultades derivadas de la necesidad de delimitar los espacios a analizar y de obtener la información para ello. En las líneas que siguen pretendemos hacer unas breves reflexiones sobre algunos de los problemas encontrados en este sentido, al plantear un estudio concreto.

En el mundo desarrollado está teniendo lugar un proceso de envejecimiento de la población que contrasta con la juventud del Tercer Mundo, habiendo, a su vez, grandes diferencias entre campo y ciudad y dentro de ésta. Al analizar la plasmación de dicho envejecimiento en el interior de la ciudad, en el caso concreto de Madrid, se observó que existía un neto contraste espacial: las áreas interiores aparecian muy envejecidas, coincidiendo con el centro histórico, frente a una periferia muy joven. Esta situación se identificaba con los resultados obtenidos en estudios similares de otras grandes ciudades europeas. En el trabajo se pudo, además, constatar de forma matemática, la existencia de una co-

\footnotetext{
* Departamento de Geografía. UNED.
} 
rrelación entre el grado de envejecimiento y la distancia al centro de la ciudad. Estos resultados movieron a plantear una ampliación del estudio en el que se trataría de analizar el fenómeno de la diferenciación espacial del envejecimiento intraurbano en toda la red urbana española, formada por las ciudades de más de 50.000 habitantes y las capitales de provincia.

La primera parte (estudio de la distribución espacial del envejecimiento en la ciudad) es el análisis de un fenómeno, que muestra la existencia de diferenciación interna y se inserta en la línea de otros múltiples estudios, cada día más abundantes, que pretenden ayudar a solucionar los problemas que surgen en el complejo mundo urbano, mediante su mejor conocimiento. En este caso, cuando se estudia un fenómeno en una sola ciudad, la cuestión básica es la delimitación de la unidad de análisis. Puede optarse por utilizar las unidades administrativas o estadisticas, que tienen la ventaja de coincidir con la información. Ahora bien, si la escala de análisis es de gran detalle, será conveniente establecer unidades propias, aplicando los criterios adecuados en función del objetivo que se pretende. (Muguruza, C. y Santos, J. M., 1989) (Calvo, J. L., 1978). En este tipo de estudios existe la posibilidad de obtener datos muy desagregados (calle, manzana...), que pueden ser reagrupados posteriormente conforme a los criterios que se establezcan. En el trabajo inicial del envejecimiento que comentábamos, resultaba útil la división en barrios de Madrid, existente en el Ayuntamiento, en cuyo marco se obtenian los datos y que permitia establecer el contraste entre centro y periferia y la diferenciación interna en los dos ámbitos.

Al acometer la segunda parte surgieron los problemas a que se hacía referencia. En primer lugar la obtención de información resulta sumamente dificultosa, ya que no se publican los datos oficiales del censo o del padrón municipal en detalle, sino tan sólo resúmenes globales de los mismos. Por tanto, cuando se precisa de información desagregada, se ha de acudir a cada Ayuntamiento.

El proceso de solicitud de dichos datos, al manejar un volumen grande de entidades, es sumamente largo y los resultados decepcionantes, ya que es prácticamente imposible obtener respuesta del $100 \%$, quedando ésta reducida, por lo general, a un 20 ó $30 \%$, en el mejor de los casos. En ocasiones simplemente no contestan a la solicitud, en otras no conceden el permiso, o manifiestan no tener mecanizado el padrón, o tenerlo entregado a una empresa privada, que cobra cifras astronómicas por facilitar los datos. Otros problemas se refieren a la obtención de planos municipales sobre los que cartografiar la información..., etc. 
No obstante, estos son problemas comunes a toda investigación. Ahora bien, en el caso citado se añaden otra serie de dificultades, que se refieren a las divisiones administrativas, objeto de estas reflexiones.

\section{LAS DIVISIONES EXISTENTES}

De acuerdo con el Reglamento de Población y Demarcación Territorial de las Entidades Locales (R.D. 169/1986, de 11 de julio) y con la Ley Reguladora de las Bases del Régimen Local (Ley 7/1985, de 2 de abril), es competencia del Ayuntamiento la división del término municipal en distritos y barrios y las variaciones de los mismos (Título primero, artículo 1. 4.). La necesidad de tales divisiones surgió subsidiariamente al planeamiento urbano de los años setenta, como las unidades básicas para la descentralización y la planificación de determinados equipamientos efectuada a propuesta de quienes realizaban los planes, a menudo sin crítica ni oposición en el Ayuntamiento.

En el capitulo II de la citada Ley en el artículo 74.1. referente al padrón municipal, dice que las hojas de inscripción padronal deberán ordenarse y numerarse correlativamente por distritos, secciones, manzanas, calles, edificios y viviendas. En el artículo 77.1. dice, también, que los ficheros de residentes se deberán ordenar por distritos, secciones y domicilios.

De lo expuesto se desprende, por tanto, que todo municipio debe estar dividido en distritos y secciones (dado que es preceptivo que de acuerdo con ellas se ordenen los datos), mientras que es potestativo el efectuar la división en barrios. Efectivamente todas las ciudades cuentan con una división en distritos y otra en secciones censales. Estas últimas resultan unidades pequeñas, que coinciden con las secciones electorales. Están basadas en un número máximo de habitantes (3.500) y un número mínimo de votantes (500), por lo que su plasmación espacial presenta una notable variedad de forma y tamaño.

El distrito es, en cambio, una unidad de grandes dimensiones, que abarca un número indeterminado de secciones, absolutamente variables de unos municipios a otros y aún dentro del mismo municipio. Hay además municipios que tienen una tercera división denominada zona estadística, de tamaño intermedio (por ejemplo, Barcelona), y otros que tienen barrios, división igualmente de tamaño intermedio entre las citadas (por ejemplo, Madrid). 
Esta diversidad de divisiones presenta, además, una enorme variedad de formas, según los criterios con que haya sido efectuada por el municipio, que la aprueba en sesión plenaria del Ayuntamiento. Así, los distritos aparecen unas veces dibujados desde el centro, o desde la zona más antigua hacia la periferia, a modo de ondas concéntricas. Otras veces son casi una cuadricula superpuesta a la trama urbana, otras son sectoriales desde el centro..., etc. Normalmente el tamaño aumenta hacia la periferia, pero tampoco siempre es asi. (vid figuras).

Con este panorama resulta muy problemático abordar un trabajo como el citado. En la ya mencionada segunda parte se pretendia analizar la diferenciación espacial intraurbana en las ciudades españolas, para saber si el fenómeno de la correlación entre envejecimiento y distancia al centro, que se daba en la capital, al igual que en otras grandes urbes europeas, existía en todas las ciudades, o si había una relación entre el fenómeno y el tamaño de la ciudad (si la correlación aparecía a partir de un determinado volumen de población). Para efectuar un estudio de estas características (u otro similar como podian ser densidades edificatorias, población activa, natalidad, etc.) utilizar como base la sección censal resulta excesivo, ya que supone un mosaico extremadamente minucioso, que no se justifica en un trabajo que abarca muchas unidades de análisis (ciudades). Por el contrario, el distrito se manifiesta como perfectamente inoperante. En primer lugar por su excesivo tamaño, por la disparidad entre unos y otros dentro de la ciudad y, sobre todo, porque su disposición no se relaciona, en general, (el caso de Madrid, Valencia, Barcelona... son excepciones) con la evolución histórica de la ciudad y no permite mostrar los contrastes entre las distintas áreas de la ciudad, tanto entre las centrales y periféricas, como era preciso en un estudio en que la distancia al centro era decisiva, como entre las meramente naturales o sociales, según el estudio de que se trate.

Por tanto, en un estudio de carácter general se percibieron las grandes dificultades que se derivan de la heterogeneidad de las divisiones administrativas de la ciudad, al tiempo que se hacia patente la necesidad de disponer de una entidad intermedia entre las existentes, surgida de la combinación de diversos criterios no meramente numéricos o geométricos.

\section{EI BARRIO COMO UNIDAD DE ANÁLISIS}

En definitiva se trata de proponer la utilización del barrio como unidad administrativa entre el distrito y la sección censal, evitando a un 
tiempo la creación de otras unidades (por ejemplo las zonas estadísticas), que contribuyen a más heterogeneidad y confusión.

El concepto de barrio es, al igual que el de la ciudad y lo urbano, muy utilizado, pero muy impreciso. Los habitantes de cada ciudad emplean con profusión el término, pero tienen enormes dificultades a la hora de definir, e incluso delimitar. En trabajos científicos es una unidad habitual, tanto en Geografia como en Sociología y otras Ciencias Sociales. En esta ocasión no se trata de definirlo, ni de precisar sus características, sino de defender su necesidad y utilidad para determinados fines.

Apenas se encuentran municipios que los tengan delimitados. No obstante, hay Ayuntamientos que sienten esta preocupación. Algunos tienen ya una división (caso de Madrid), en la que se han respetado barrios antiguos, que tienen su entidad y en la que sus habitantes se sienten identificados y se han trazado otros barrios nuevos, con mayor o menor fortuna. En el caso citado se realizaron por el área de urbanismo e Infraestructura (AUI) del Ayuntamiento estudios conducentes a la fijación de criterios para la delimitación (históricos, demográficos y urbanísticos). Hay otros, como Bilbao, que incluso han publicado su preocupación y los criterios que les han movido a dividir su municipio en barrios:

“...Esta división, que considera cuatro unidades territoriales diferentes: Módulos y Secciones (unidades básicas de información a nivel municipal), Barrios y Distritos, constituye el primer paso para la puesta en marcha de un nuevo proyecto municipal: el proyecto de la descentralización y participación ciudadana, cuyo objetivo es acercar la administración a los ciudadanos, aumentar y mejorar los servicios y reducir las desigualdades existentes entre las diferentes zonas en cuanto a dotación de equipamientos... Los distritos jugarán un papel fundamental, no sólo en el proceso de descentralización (para el que se necesitan ámbitos territoriales grandes) y participación ciudadana ( para el que se necesitan unidades territoriales pequeñas), sino también en la planificación de equipamientos (para el que se necesitan diferentes ámbitos territoriales según el tipo de equipamiento)..." Por eso: “... se decidió considerar diferentes unidades territoriales distritos y barrios...".

“... los barrios serán las unidades territoriales básicas para la participación ciudadana y la planificación de determinados equipamientos.

Son unidades territoriales menores, más homogéneas y con intereses comunes, en las que se establecen relaciones directas entre la gente de conocimiento o frecuentación y se manifiesta un fuerte sentido de pertenencia a las mismas.

Para la localización de los barrios se han tenido en cuenta fundamentalmente el criterio asociativo: las asociaciones existentes y su ámbito de actuación, imponiéndose una única restricción: un nivel poblacional superior a 1.000 habitantes. 
Así han quedado delimitados un gran número de barrios, recurriéndose a criterios históricos, urbanísticos o sociológicos en el resto de los casos hasta llegar a la delimitación de un total de 34 barrios y 8 zonas de deseminado en las que se incluyen el espacio no urbanizado, los edificios dispersos y aislados y las zonas de futura habilitación especial no incluidas en barrios concretos...".

(Ayuntamiento de Bilbao, 1987)

Desde hace mucho tiempo se realizan interesantísimos trabajos de investigación centrados en la ciudad, por parte de un rico colectivo de profesionales de diversos campos: geógrafos, urbanistas, arquitectos, sociológos... En muchos de ellos se busca analizar la estructura urbana, en la que subyace un mosaico de mundos sociales (TIMMS, D. 1976), centrados en la diferenciación residencial y segregación social. Sin entrar aquí a analizar ni las características de tales estudios, ni su valoración, utilidad, etc., si creemos que es conveniente apoyar un intento de coordinación, de modo que los Ayuntamientos, que cada día cuentan con mayor número de especialistas en los campos citados, no sólo realizaran sus propias investigaciones, sino que utilizaran el trabajo de tantos investigadores que estudian la realidad urbana y cuyas conclusiones la mayor parte de las veces no sobrepasan el nivel académico, perdiéndose en el olvido más absoluto.

Establecer en las ciudades unas divisiones de tamaño medio, que englobaran las secciones censales con fines electorales, pero en las que se consideraran criterios diversos y factores influyentes, buscando las áreas naturales de Zorbaugh, o algo similar ¿no ayudaría a mejor planificar y, en definitiva, a mejor administrar? Esta opinión parece coincidir con la manifestada por los Ayuntamientos de Madrid y Bilbao.

Los Ayuntamientos, que tienen en sus manos toda la información llegarían a un mejor conocimiento de su propio ser y facilitarian, al agrupar aquella información en unidades "lógicas", establecidas con criterios múltiples y semejantes para todas las ciudades, el acceso por parte de los investigadores a una información más homogénea y de fácil manejo para estudios de carácter general, como el del ejemplo que ha motivado estas líneas, que pretenden llamar la atención sobre. la conveniencia y necesidad de realizar esta división para todos los Ayuntamientos (a partir de un determinado tamaño de población) y fomentar la coordinación de la investigación científica y la Administración, lográndose, en definitiva una mejor gestión. 
Contraste entre tamaño y densidad de población entre las distintas zonas estadísticas y distritos del municipio de Barcelona.

2. DISTRICTES MUNICIPALS

2.2. Superficie i densitat 1986

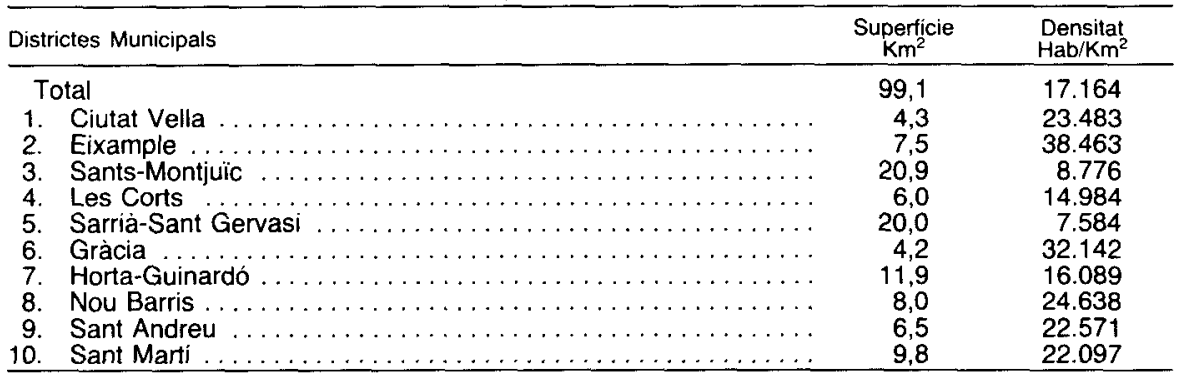

Font: Servei d'Estadistica. Ajuntament de Barcelona.

ZONES ESTADÍSTIQUES

3.2. Superifície i densitat 1986

\begin{tabular}{|c|c|c|}
\hline Zones Estadístiques & $\begin{array}{c}\text { Superticie } \\
\mathrm{Km}^{2}\end{array}$ & $\begin{array}{c}\text { Densitat } \\
\mathrm{Hab} . / \mathrm{Km}^{2}\end{array}$ \\
\hline 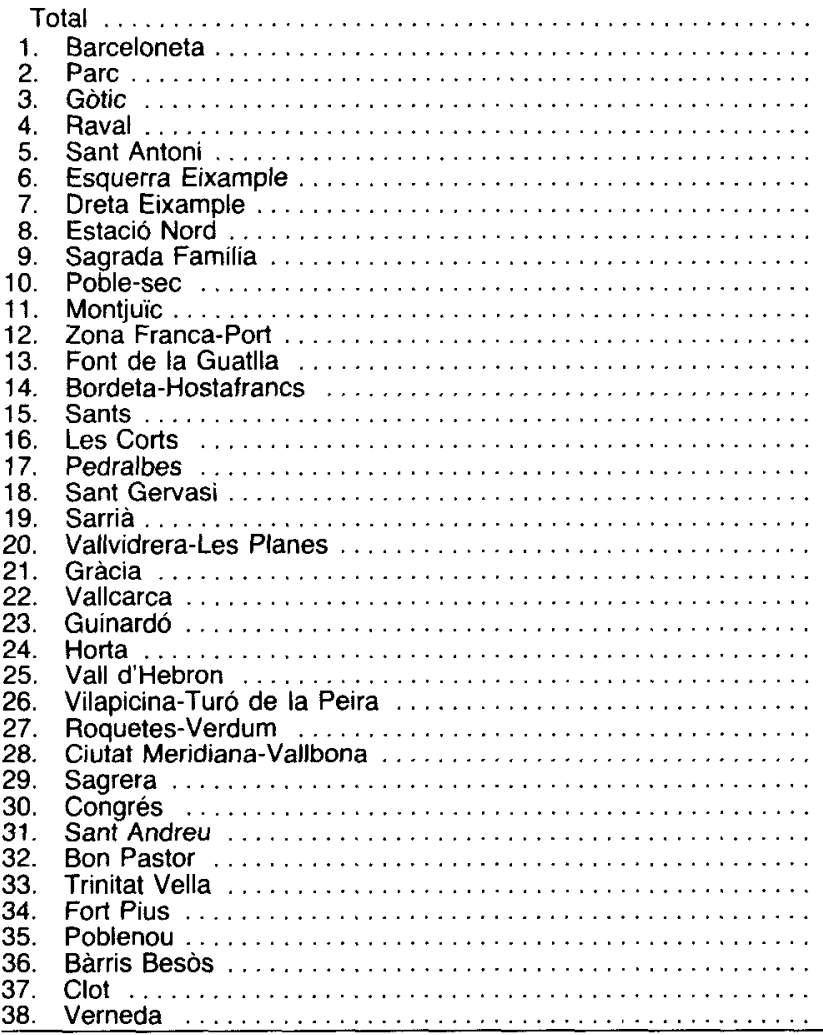 & $\begin{array}{r}99,15 \\
1,27 \\
1,13 \\
0,81 \\
1,10 \\
0,81 \\
2,56 \\
2,12 \\
0,96 \\
1,05 \\
0,74 \\
5,08 \\
12,14 \\
0,45 \\
0,59 \\
1,89 \\
3,12 \\
2,86 \\
5,19 \\
4,39 \\
10,45 \\
2,04 \\
2,11 \\
2,09 \\
4,07 \\
5,78 \\
1,94 \\
2,98 \\
3,09 \\
1,44 \\
0,40 \\
1,79 \\
2,08 \\
0,82 \\
0,43 \\
5,01 \\
1,06 \\
1,43 \\
1,88 \\
\end{array}$ & $\begin{array}{r}17.164 \\
14.091 \\
21.164 \\
21.536 \\
38.190 \\
52.048 \\
43.818 \\
22.508 \\
32.617 \\
52.524 \\
52.392 \\
338 \\
2.359 \\
21.416 \\
33.215 \\
44.917 \\
24.065 \\
5.100 \\
21.946 \\
8.200 \\
190 \\
48.328 \\
16.595 \\
37.093 \\
20.083 \\
5.668 \\
39.200 \\
35.531 \\
4.951 \\
38.755 \\
40.400 \\
29.021 \\
6.827 \\
11.537 \\
26.914 \\
9.342 \\
27.201 \\
43.737 \\
35.670 \\
\end{array}$ \\
\hline
\end{tabular}

Font: Servei d'Estadística. Ajuntament de Barcelona. 


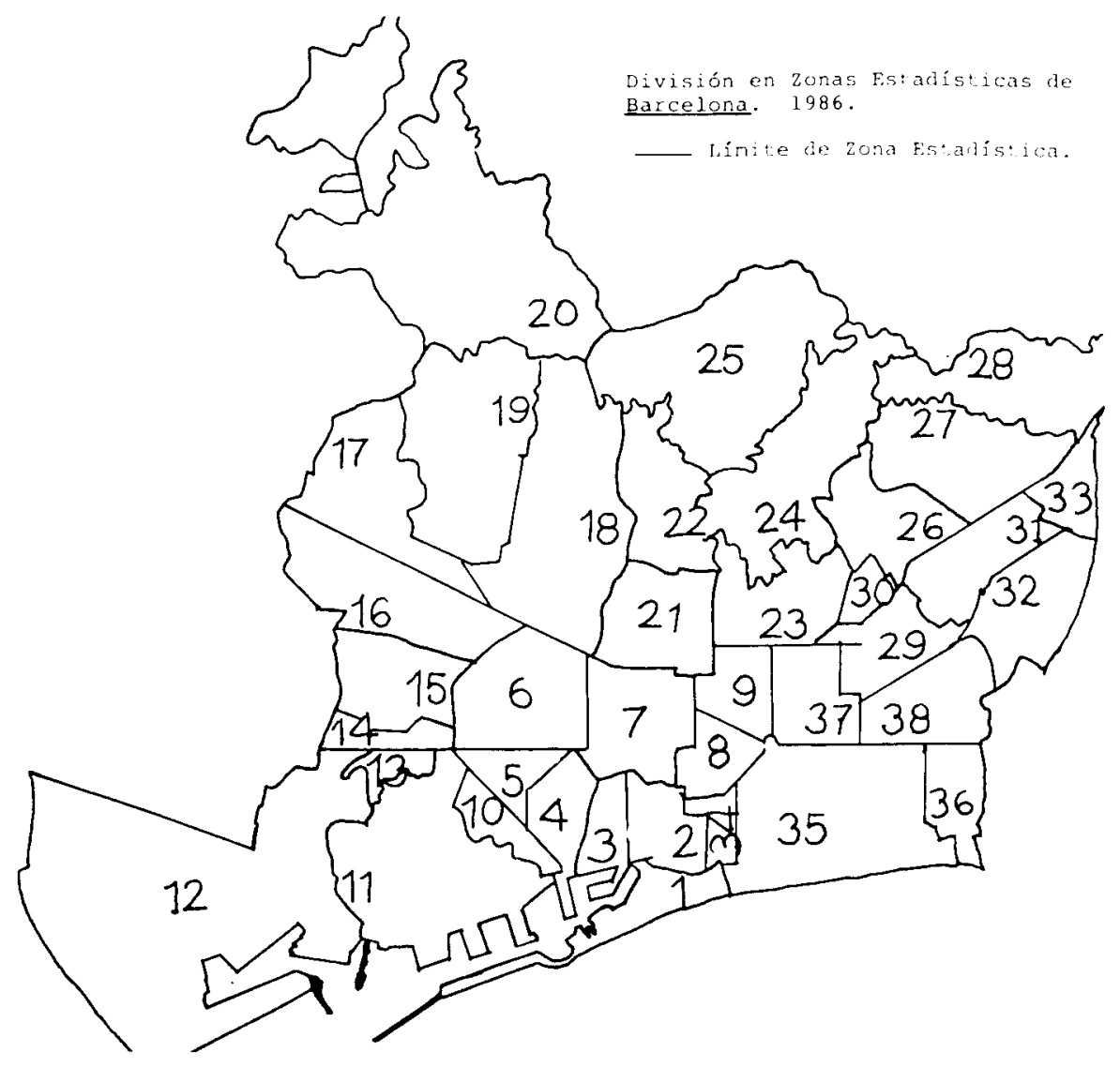




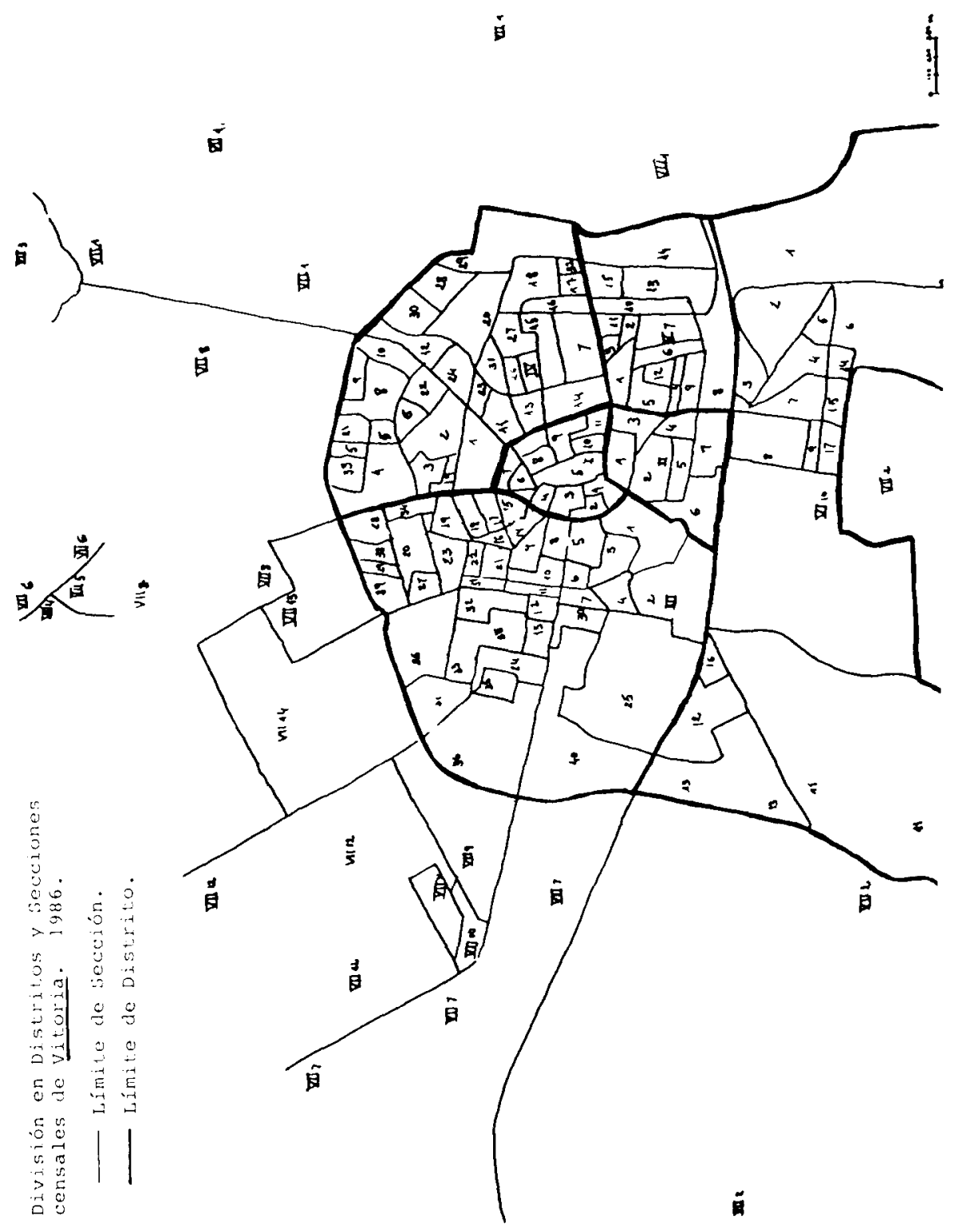




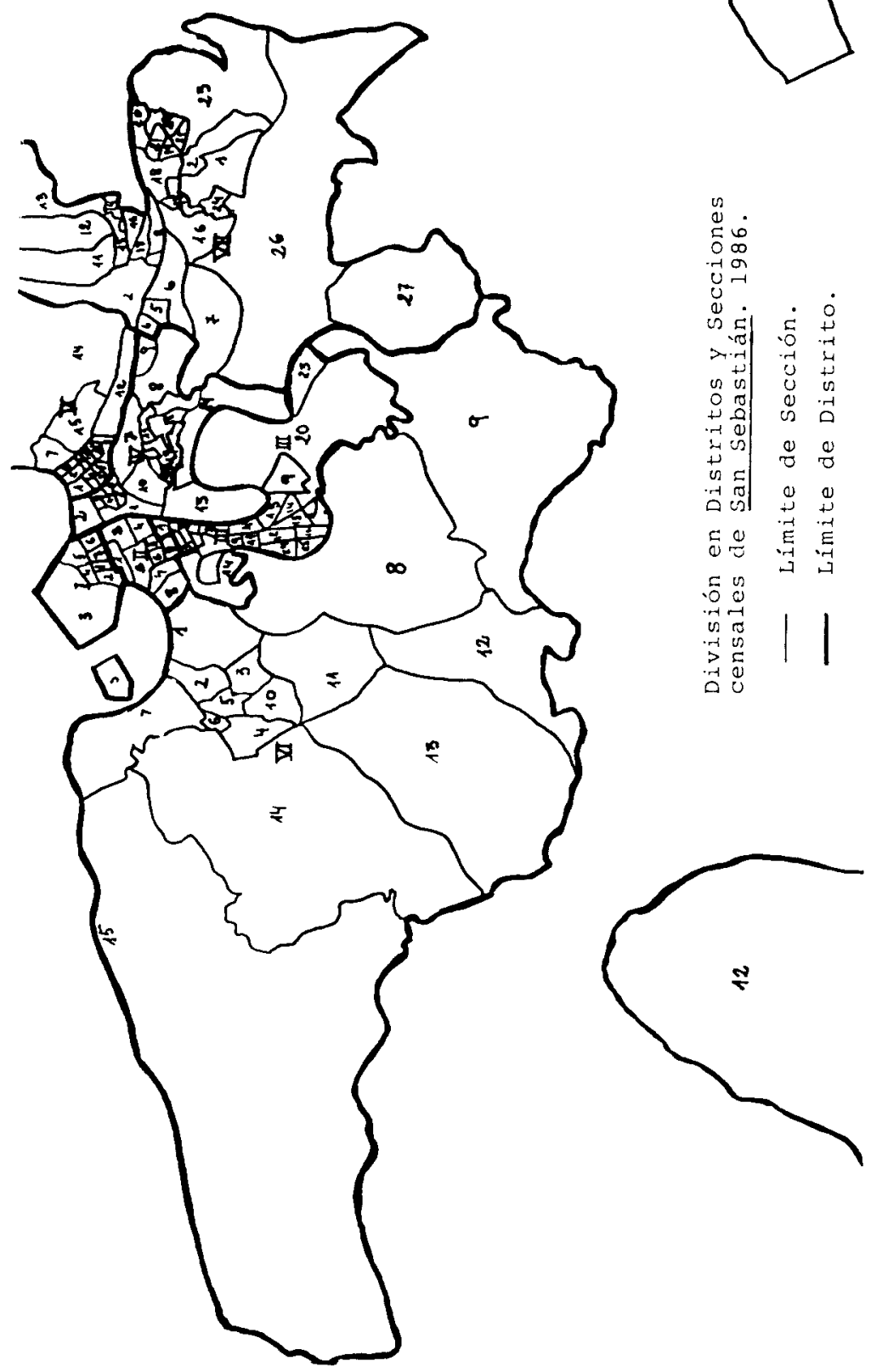


División en Barrios de Badalona. 1986.

-.-- Limite de Barrio.

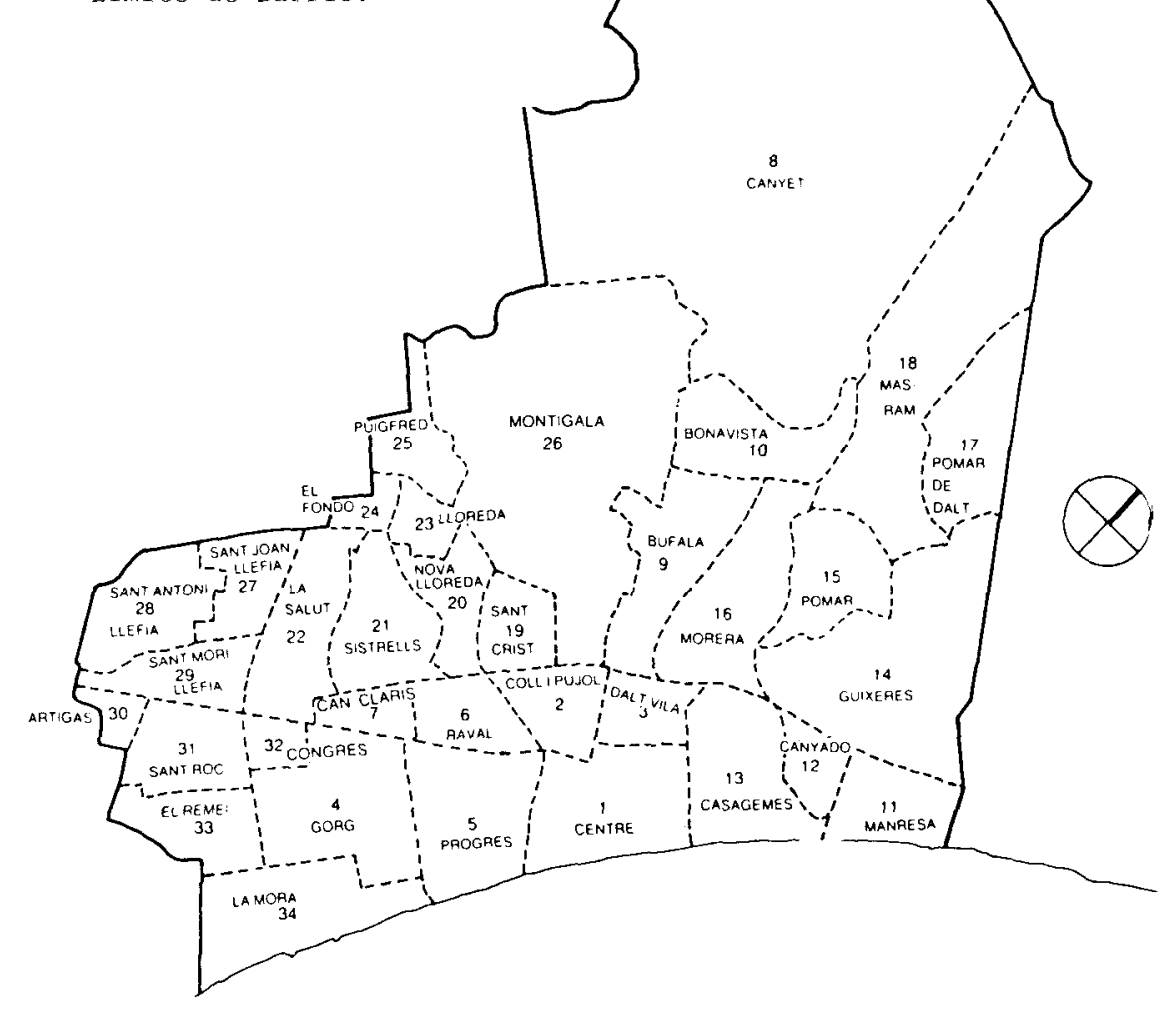




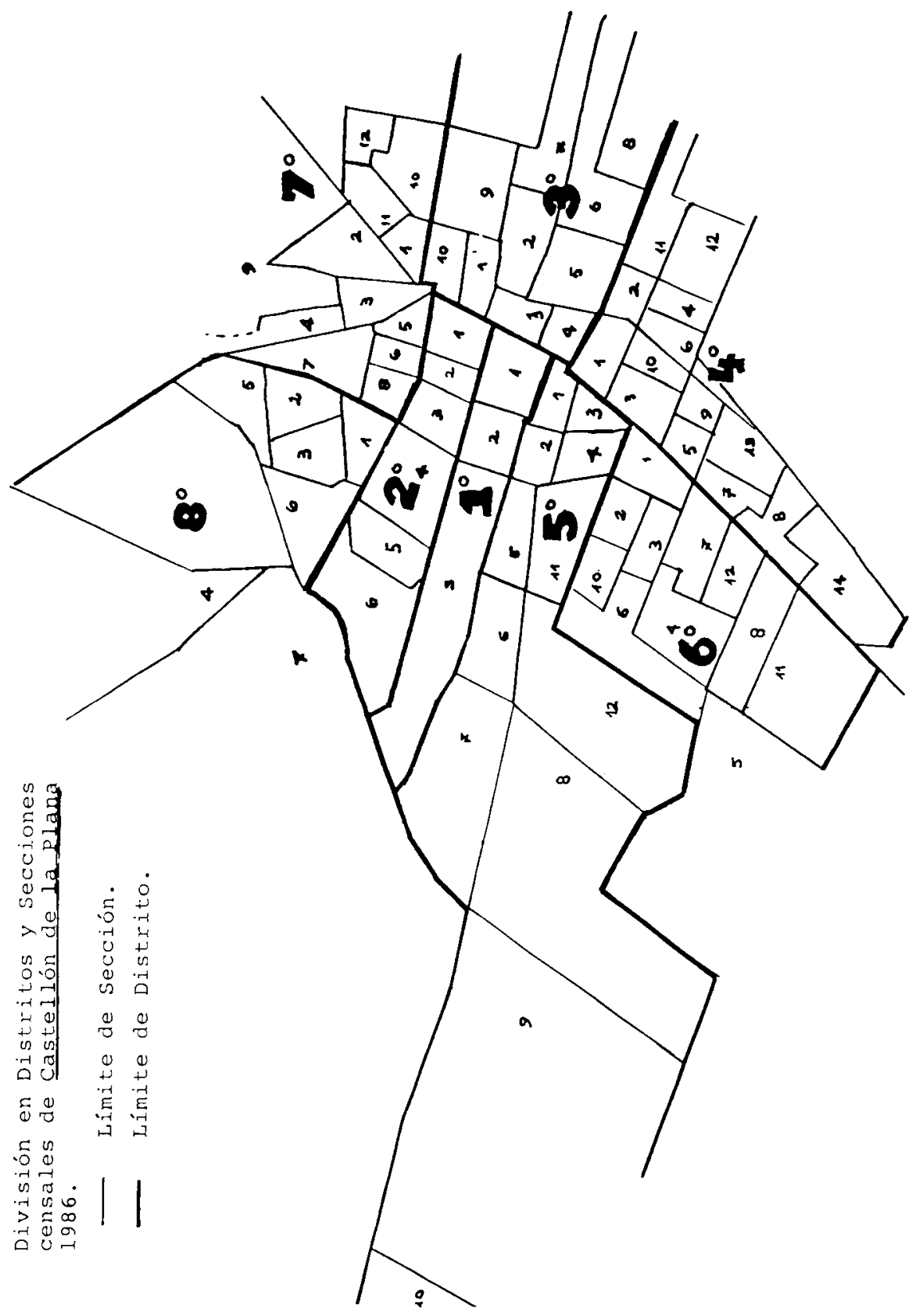




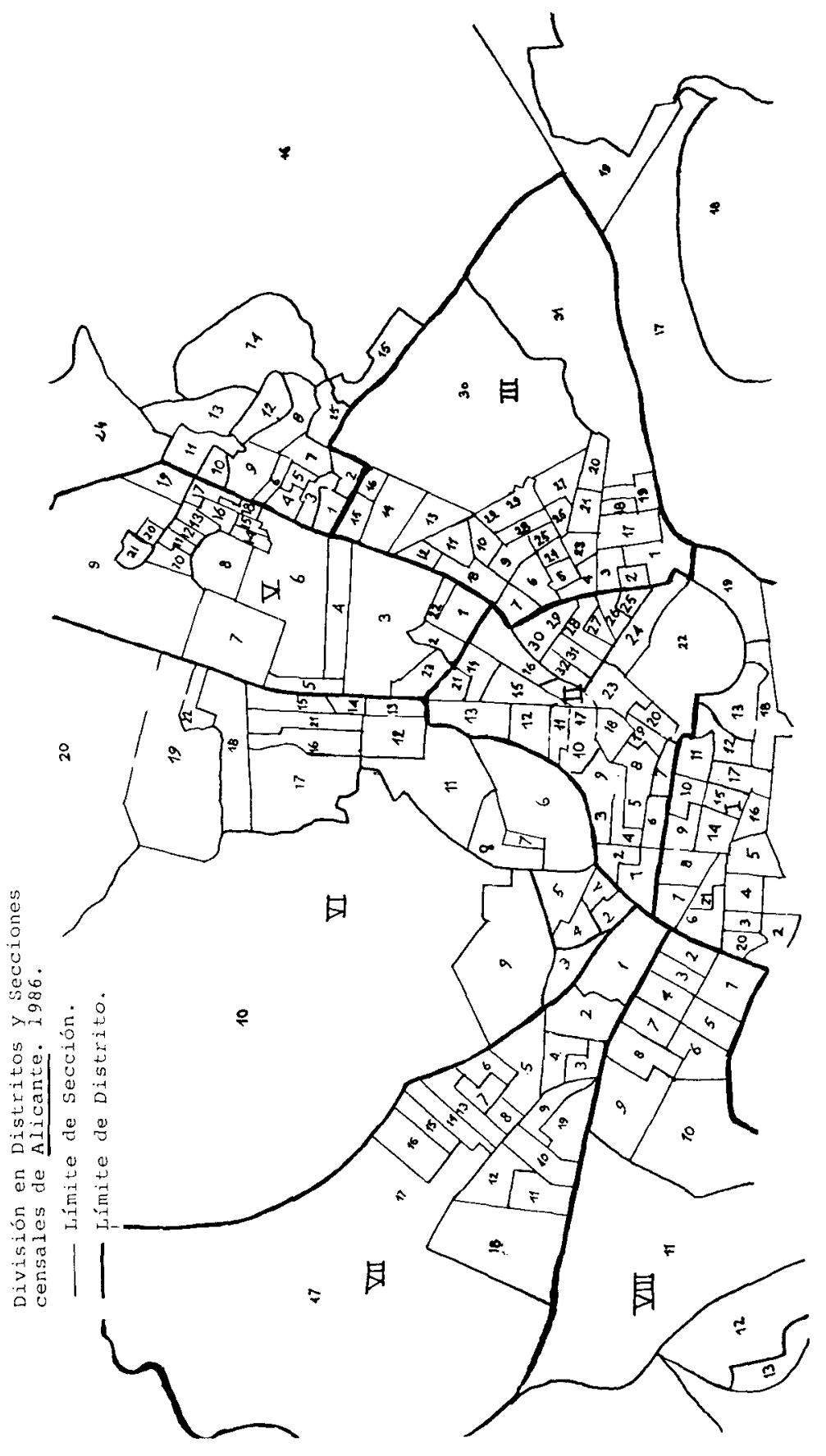




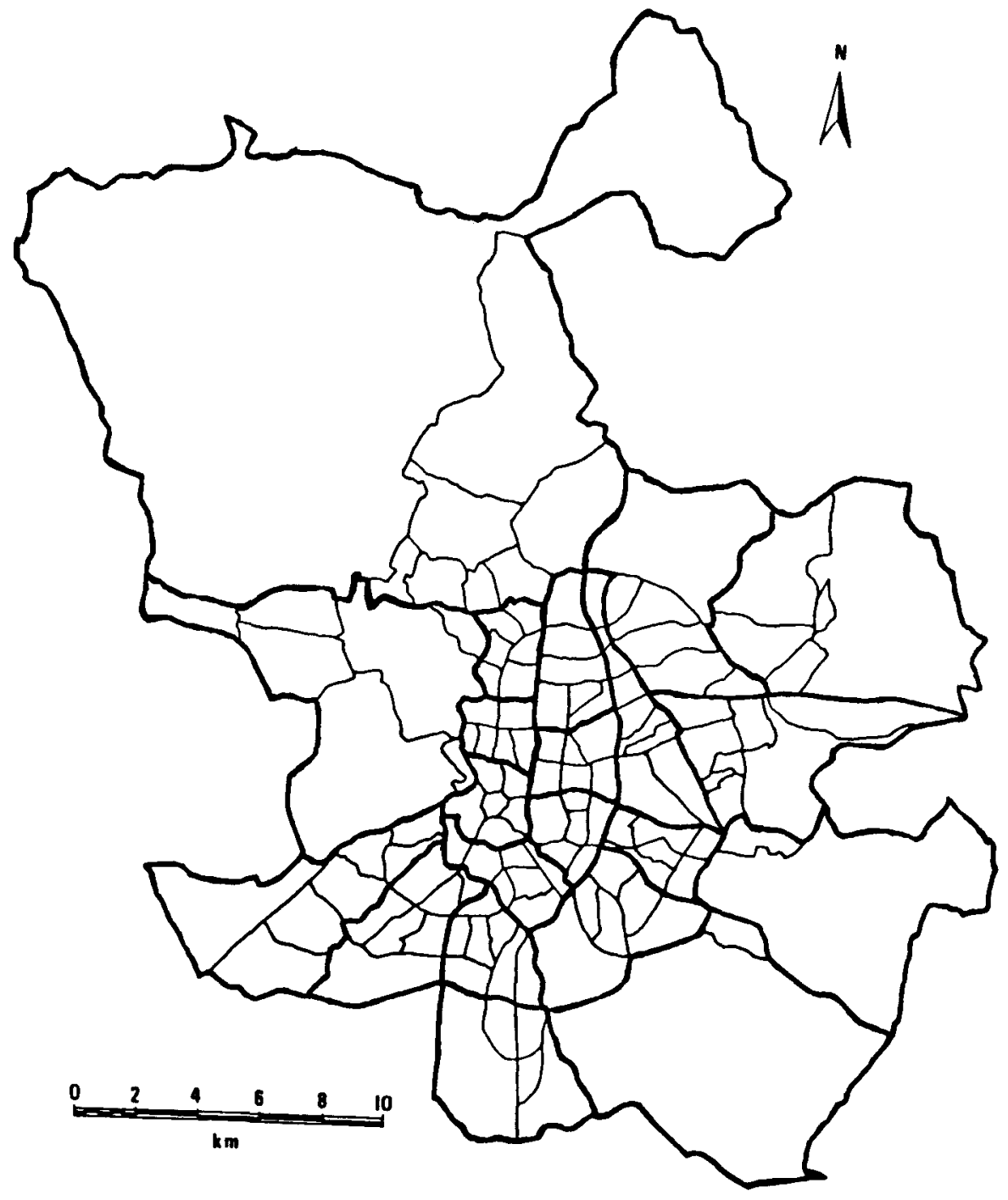

División en Distritos y Barrios de Madrid. 1988.

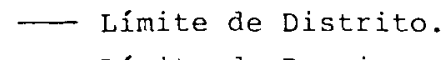

- Limite de Barrio. 


\section{BIBLIOGRAFIA}

Calvo Palacios, J. L. 1980: "Unidades de análisis y densidades urbanas: Zaragoza", Geographicalia núm. 5, Enero-Marzo. Zaragoza, págs. 5-32.

CONSULTOR DE LOS AYUNTAMIENTOS, El. 1987: “Legislación local. Textos legales concordados y anotados del Régimen Local». Tomo I. El Consultor de los Ayuntamientos y de los Juzgados. Madrid, 830 págs.

Muguruza Cañas, C. y Santos Preciados, J. M. 1989: "La importancia de las unidades de análisis en el modelo de ecologia factorial", Espacio, Tiempo y Forma. Serie VI, núm. 2, págs. 87-101.

IsURI, R. 1987: "Bilbao ocho distritos". Bilbao 2. Ayuntamiento de Bilbao. Área de Relaciones Ciudadanas y Descentralización, Diciembre 1987, págs. 12-13.

TIMms, D. 1976: «El mosaico urbano». Madrid, IEAL, 458 págs. 